\title{
DENSITIES AND DIFFERENTIABILITY PROPERTIES OF GAUSS SEMIGROUPS ON A LIE GROUP
}

\author{
EBERHARD SIEBERT
}

\begin{abstract}
We consider an absolutely continuous Gauss semigroup on a connected Lie group. Integrability and boundedness properties for the corresponding densities are established. Moreover it is shown that the Gauss measures transform integrable functions into infinitely differentiable solutions of certain partial differential equations. Finally, we prove that the semigroup acts on many Banach spaces as a differentiable operator semigroup.
\end{abstract}

Introduction. In [11] we characterized the absolute continuity of a Gauss semigroup $\left(\mu_{t}\right)_{t>0}$ on a Lie group $G$ by means of its generator $N$. Moreover, we established some differentiability properties of the densities $v_{t}$ of the measures $\mu_{t}$. In this paper we continue these investigations. Our results rely on those of [11]. In particular, the hypoellipticity of the differential operator $N-\partial / \partial t$ on the manifold ] $0, \infty[\times G$ plays a crucial role. In fact, if $N-\partial / \partial t$ is even elliptic then our subject has quite a long history, starting in 1959 with Nelson's paper [7] (cf. the discussion in $[2, \mathrm{R} 6.3])$. The new element appearing in this context is hypoellipticity.

Our Theorem 1 establishes some integrability and boundedness properties of the densities $v_{t}$. Here the important point is that the densities turn out to be square integrable. In particular this gives pointwise estimations. Theorem 2 yields that the absolutely continuous Gauss measures $\mu_{t}$ transform $L^{p}(G)$-functions into infinitely differentiable functions that solve the partial differential equation $N=\partial / \partial t$ on ] $0, \infty[\times G$. Theorem 3 shows that many Banach space representations of $G$ turn the absolutely continuous Gauss semigroup $\left(\mu_{t}\right)_{t>0}$ into a differentiable operator semigroup. This is a necessary condition for the semigroup $\left(\mu_{t}\right)_{t>0}$ to be holomorphic (roughly speaking this means that $\mu_{t}$ depends holomorphically on $t$ ). It is well known that certain classes of Gauss semigroups are holomorphic (cf. $[5,6,10,12])$. But there is still the conjecture (supported by Theorem 3 ) that every absolutely continuous Gauss semigroup is holomorphic. ${ }^{1}$

Preliminaries. By $\mathbf{R}_{+}^{*}$ we denote the set of positive real numbers. A real valued function $f$ defined on $\mathbf{R}_{+}^{*}$ is said to be locally bounded if $\sup \{|f(t)|: 1 / s \leqslant t \leqslant s\}<\infty$ for all $s \in \mathbf{R}_{+}^{*}$.

Received by the editors April 27, 1982 and, in revised form, February 28, 1983.

1980 Mathematics Subject Classification. Primary 60B15; Secondary 43A05, 47F05.

Key words and phrases. Gauss semigroup, connected Lie group, absolute continuity, hypoelliptic differential operator, weakly sequentially complete Banach space, differentiable operator semigroup.

${ }^{\prime}$ The conjecture formulated in the introduction has been recently disproved by $\mathrm{M}$. McCrudden and R. W. Wood. 
If $M$ is a real analytic manifold we denote by $e^{\infty}(M)$ the space of real valued functions on $M$ which are differentiable infinitely often. $\mathscr{D}(M)$ is the subspace of functions in $e^{\infty}(M)$ that have compact support.

By $G$ we always denote a connected Lie group of dimension $n \geqslant 1$. Let $d$ be a left invariant Riemannian metric on $G$. We put $\tau(x)=d(x, e), x \in G$, where $e$ is the identity of $G$. Let $g$ be the Lie algebra of $G$ and exp the exponential mapping of $\mathfrak{g}$ into $G$. Every $X \in \mathfrak{g}$ defines a differential operator $\tilde{X}$ on $G$ by

$$
\tilde{X} f(x)=\lim _{t \downarrow 0} t^{-1}\{f(x \exp t X)-f(x)\}
$$

for all $f \in e^{\infty}(G)$ and $x \in G$.

By $L(G)$ or $L^{\infty}(G)$ we denote the Banach space of bounded real valued Borel measurable functions $f$ on $G$ with the norm $|f|_{\infty}=\sup \{|f(x)|: x \in G\}$. Let $\mathrm{C}^{b}(G)$ $=\{f \in L(G): f$ is continuous $\}, e^{0}(G)=\left\{f \in e^{b}(G): f\right.$ vanishes at infinity $\}$, and $\mathcal{K}(G)=\left\{f \in \bigodot^{0}(G): f\right.$ has compact support $\}$. Moreover we put $\mathscr{E}(G)=\bigodot^{\infty}(G) \cap$ $e^{b}(G)$. If $f$ is a function on $G$ and if $x \in G$, we define functions ${ }_{x} f, f_{x}, f^{*}$ by ${ }_{x} f(y)=f(x y), f_{x}(y)=f(y x), f^{*}(y)=f\left(y^{-1}\right)$ (all $\left.y \in G\right)$. By $\lambda$ and $\rho$ we denote a left and a right Haar measure on $G$, respectively, connected by $\rho=\Delta \cdot \lambda$ where $\Delta$ is the modular function of $G$.

Let $L^{p}(G)$ be the Banach space of (equivalence classes of) real valued Borel measurable functions $f$ on $G$ such that $|f|^{p}$ is $\lambda$-integrable and let $|f|_{p}=\left\{\int|f|^{p} d \lambda\right\}^{1 / p}$ $(1 \leqslant p<\infty)$. $\mathfrak{M}^{1}(G)$ denotes the space of probability measures on the $\sigma$-algebra of Borel sets in $G$. With every $\mu \in \mathfrak{T}^{1}(G)$ there is associated a contraction operator $T_{\mu}$ on $L(G)$ defined by $\left(T_{\mu} f\right)(x)=\int f(x y) \mu(d y)$ for all $x \in G$ and $f \in L(G)$.

A family $\left(\mu_{t}\right)_{t>0}$ in $\Re^{\prime}(G)$ is said to be a Gauss semigroup if no $\mu_{t}$ is a point measure, if $\mu_{s} * \mu_{t}=\mu_{s+t}$ for all $s, t \in \mathbf{R}_{+}^{*}$ (where $*$ denotes convolution in $\mathfrak{T}^{1}(G)$ ), and if $\lim _{t \downarrow 0} t^{-1} \mu_{t}(\mathbf{C} U)=0$ for every open neighborhood $U$ of the identity $e$ of $G$. The semigroup $\left(\mu_{t}\right)_{t>0}$ is said to be absolutely continuous if every measure $\mu_{t}$ is absolutely continuous with respect to $\lambda$ (or $\rho$ ).

As is well known, for every $f \in \mathcal{E}(G)$ there exists

$$
A(f)=\lim _{t \downarrow 0} \frac{1}{t}\left\{\int f d \mu_{t}-f(e)\right\} .
$$

$A$ is called the generating functional of the semigroup $\left(\mu_{t}\right)_{t>0}$. Moreover there exist elements $X_{0}, X_{1}, \ldots, X_{r}$ of $\mathrm{g}(r \leqslant n)$ such that the differential operator $N=\tilde{X}_{0}+$ $\tilde{X}_{1} \tilde{X}_{1}+\cdots+\tilde{X}_{r} \tilde{X}_{r}$ on $G$ represents $A$ in the sense that $N f(x)=A\left({ }_{x} f\right)$ for all $x \in G$ and $f \in \mathcal{E}(G)$. (For more relevant information on Gauss semigroups cf. [2, 11].) We shall repeatedly apply the following two results:

TheOREM A. Let $\left(\mu_{t}\right)_{t>0}$ be a Gauss semigroup on $G$. Then for every $s \in \mathbf{R}_{+}^{*}$, there exists a $c \in \mathbf{R}_{+}^{*}$ such that

$$
\sup \left\{\int \exp \left(c \tau(x)^{2}\right) \mu_{t}(d x): 0<t \leqslant s\right\}<\infty .
$$


In particular, for every $b \in \mathbf{R}_{+}^{*}$, the function

$$
t \rightarrow \int \exp (b \tau(x)) \mu_{t}(d x)
$$

is locally bounded on $\mathbf{R}_{+}^{*}(c f .[6$, Theorem 2]).

THEOREM B. Let $\left(\mu_{t}\right)_{t>0}$ be an absolutely continuous Gauss semigroup on G. Let $f \in L(G)$ and put $w(t, x)=T_{\mu_{t}} f(x)$ for all $(t, x) \in \mathbf{R}_{+}^{*} \times G$. Then the function $w$ is in $e^{\infty}\left(\mathbf{R}_{+}^{*} \times G\right)$ and satisfies $\partial w(t, x) / \partial t=N w(t, x)$ for all $(t, x) \in \mathbf{R}_{+}^{*} \times G[11$, Theorem 3].

Results. Let us now fix once and for all an absolutely continuous Gauss semigroup $\left(\mu_{t}\right)_{t>0}$ on the Lie group $G$ with generating functional $A$ and representing differential operator $N$. Then by Theorems 1 and 2 in [11] there exists an infinitely differentiable function $\vartheta \geqslant 0$ on $\mathbf{R}_{+}^{*} \times G$ such that $v_{t}=\vartheta(t, \cdot)$ is a $\lambda$-density of the measure $\mu_{t}$.

Lemma. Let $p \in\left[1, \infty\left[\right.\right.$ and $f \in L^{p}(G)$ such that $f \geqslant 0$. Then for every $t \in \mathbf{R}_{+}^{*}$ the integral $\mu_{t} * f(e)=\int f\left(y^{-1}\right) \mu_{t}(d y)$ is defined and finite. Moreover the function $t \rightarrow$ $\mu_{t} * f(e)$ is locally bounded on $\mathbf{R}_{+}^{*}$.

Proof. Let $C=\mathbf{R}_{+}^{*} \times G$. Now $u(t, x)=\mu_{t} * f(x)$ is defined for all $(t, x) \in C$ (since $f \geqslant 0$ ). Moreover, $u(t, x)=\sup \left\{\mu_{t} * \min (f, n)(x): n \geqslant 1\right\}$ implies the measurability of the function $u$ (cf. Theorem B).

Now we proceed as in the proofs of Theorems 1 and 3 in [11]. We shall only give the arguments. By

$$
\varphi(h)=\int\left(\int u\left(t, x^{-1}\right) h(t, x) \rho(d x)\right) d t \quad(h \in \mathscr{D}(C))
$$

there is defined a distribution $\varphi$ on the manifold $C$. Now $P=N-\partial / \partial t$ is a differential operator on $C$. One easily shows $P \varphi=0$. Since the Gauss semigroup $\left(\mu_{t}\right)_{t>0}$ is absolutely continuous, $P$ is hypoelliptic [11, Corollary of Theorem 2]. Consequently there exists a function $v \in e^{\infty}(C)$ such that $v(t, x)=u\left(t, x^{-1}\right)$ for almost all $(t, x) \in C$ and such that $P v=0$.

Taking into account Fatou's lemma we conclude $u(t, x) \leqslant v\left(t, x^{-1}\right)$ for all $(t, x)$ $\in C$. In particular we have $\mu_{t} * f(e)=u(t, e) \leqslant v(t, e)$. Hence the assertion.

THEOREM 1. The densities $v_{t}$ of the Gauss measures $\mu_{t}$ admit the following properties:

(i) For every $b \in \mathbf{R}_{+}^{*}$ one has $e^{b \tau} v_{t} \in L^{p}(G), 1 \leqslant p \leqslant \infty$, and $e^{b \tau} v_{t} \in \bigodot^{0}(G)$ (all $t \in \mathbf{R}_{+}^{*}$ ).

(ii) For every $b \in \mathbf{R}_{+}^{*}$ the function $t \rightarrow\left|e^{b \tau} v_{t}\right|_{p}$ is locally bounded on $\mathbf{R}_{+}^{*}$ (all $p \in[1, \infty])$.

(iii) For every interval $[\alpha, \beta]$ in $\mathbf{R}_{+}^{*}$ and for every compact subset $C$ of $G$ the function $\sup \left\{{ }_{x}\left(v_{t}\right)_{y}: x, y \in C ; \alpha \leqslant t \leqslant \beta\right\}$ is $\lambda$-integrable and vanishes at infinity.

(iv) Assertions (i)-(iii) are also valid with $v_{t}^{*}$ instead of $v_{t}$.

(v) $v_{s} * v_{t}(x)=v_{s+t}(x)$ for all $x \in G$ and $s, t \in \mathbf{R}_{+}^{*}$.

PROof. Let $b \in \mathbf{R}_{+}^{*}$ be fixed. Moreover there exist constants $a, k^{*} \in \mathbf{R}_{+}^{*}$ such that

$$
k^{-1} e^{-a \tau} \leqslant \Delta \leqslant k e^{a \tau} \quad[3, \text { Proposition 1.2] }
$$


1. We first prove (i) and (ii) for $p=2$ : Let $c=a+b$. By Theorem A we have $e^{c \tau} v_{t} \in L^{1}(G)$. This yields, in view of [1, (20.2)], $f=v_{t}^{*} e^{c \tau} \Delta^{-1} \in L^{1}(G)$. Moreover, $f \geqslant 0$. Taking into account $(*)$ we obtain

$$
\begin{aligned}
\mu_{t} * f(e) & =\int f\left(y^{-1}\right) \mu_{t}(d y)=\int f\left(y^{-1}\right) v_{t}(y) \lambda(d y) \\
& =\int v_{t}(y) v_{t}(y) e^{c \tau(y)} \Delta(y) \lambda(d y) \geqslant k^{-1} \int e^{b \tau} v_{t}^{2} d \lambda .
\end{aligned}
$$

Hence the assertion in view of the lemma above.

2. Taking into account $(*)$ and part 1 we can conclude $v_{t}^{2} e^{2 b \tau} \Delta^{-1} \in L^{1}(G)$. Thus $\left(v_{t}^{*}\right)^{2} e^{2 b \tau} \in L^{1}(G)$ and $\left|v_{t}^{*} e^{b \tau}\right|_{2}=\left|v_{t} e^{b \tau} \Delta^{-1 / 2}\right|_{2}$; in particular $t \rightarrow\left|v_{t}^{*} e^{b \tau}\right|_{2}$ is locally bounded on $\mathbf{R}_{+}^{*}[\mathbf{1},(20.2)]$. Hence $v_{s} * v_{t} \in \bigodot^{0}(G)[\mathbf{1},(20.16)]$; therefore (v) is proved.

3. For fixed $t \in \mathbf{R}_{+}^{*}$ let $g=v_{t} e^{b \tau}$. As just proved we have $g, g^{*} \in L^{2}(G)$. Consequently $h=g * g \in e^{0}(G)$. In view of (v) and $\tau(x y)+\tau(y) \geqslant \tau(x)$ (all $x, y \in G)$, we have for all $x \in G$ :

$$
\begin{aligned}
h(x) & =\int v_{t}(x y) e^{b \tau(x y)} v_{t}\left(y^{-1}\right) e^{b \tau(y)} \lambda(d y) \\
& \geqslant e^{b \tau(x)} \int v_{t}(x y) v_{t}\left(y^{-1}\right) \lambda(d y)=e^{b \tau(x)} v_{2 t}(x)
\end{aligned}
$$

Consequently, $e^{b \tau} v_{2 t} \in e^{0}(G)$. Moreover,

$$
\left|e^{b \tau} v_{2 t}\right|_{\infty} \leqslant|h|_{\infty}=|g * g|_{\infty} \leqslant|g|_{2}\left|g^{*}\right|_{2}=\left|v_{t} e^{b \tau}\right|_{2}\left|v_{t}^{*} e^{b \tau}\right|_{2} .
$$

As shown above (cf. 1 and 2), the functions $t \rightarrow\left|v_{t} e^{b \tau}\right|_{2}$ and $t \rightarrow\left|v_{t}^{*} e^{b \tau}\right|_{2}$ are locally bounded on $\mathbf{R}_{+}^{*}$; hence the same is true for the function $t \rightarrow\left|v_{t} e^{b \tau}\right|_{\infty}$. Now for every $p \in] 1, \infty[$ we have

$$
\begin{aligned}
\left|v_{t} e^{b \tau}\right|_{p}^{p} & =\int e^{p b \tau} v_{t}^{p} d \lambda=\int\left\{e^{(p-1) b \tau} v_{t}^{p-1}\right\} e^{b \tau} v_{t} d \lambda \\
& \leqslant\left|v_{t} e^{b \tau}\right|_{\infty}^{p-1}\left|v_{t} e^{b \tau}\right|_{1} .
\end{aligned}
$$

Taking into account Theorem $\mathrm{A}$, thus (i) and (ii) are proved for all $p \in[1, \infty]$.

4. For the proof of (iii) we observe that there exists a constant $c \in \mathbf{R}_{+}^{*}$ such that $e^{-c \tau}$ is a $\lambda$-integrable function [3, Proposition 1.5]. In view of (ii) there exists a $\gamma \in \mathbf{R}_{+}^{*}$ such that $v_{t} \leqslant \gamma e^{-c \tau}$ for $t \in[\alpha, \beta]$. With $d=\sup \{\tau(x): x \in C\}$ we obviously have $\tau(x z y) \geqslant \tau(z)-2 d$ and, consequently, $v_{t}(x z y) \leqslant \gamma e^{2 c d} e^{-c \tau(z)}$ for all $z \in G$ and $x, y \in C$. This shows (iii).

5. Finally the proof of (iv) can now be carried out very easily by the same procedure as in the case $p=2$ considered in 2 .

Remarks. 1. By Theorem 1(i) there exists for all $b, t \in \mathbf{R}_{+}^{*}$ a $\gamma \in \mathbf{R}_{+}^{*}$ such that $v_{t} \leqslant \gamma e^{-b \tau}$.

Taking into account the first part of Theorem A, assertions (i) and (ii) of Theorem 1 also hold with $\tau^{2}$ instead of $\tau$ if the constants $b \in \mathbf{R}_{+}^{*}$ are chosen sufficiently small. 
2. For a symmetric absolutely continuous Gauss semigroup (i.e. $X_{0}=0$ ) and for $p=2$, property (i) of Theorem 1 has been asserted by P. E. T. Jørgensen [4, proof of Proposition 3.3].

Theorem 2. Let $p \in[1, \infty]$ and $f \in L^{p}(G)$. Then for every pair $(t, x) \in \mathbf{R}_{+}^{*} \times G$ the integrals

$$
u(t, x)=\mu_{t} * f(x)=\int f\left(y^{-1} x\right) \mu_{t}(d y)
$$

and

$$
w(t, x)=\int f(x y) \mu_{t}(d y)
$$

are defined and finite. Moreover $u$ and $w$ are in $\bigodot^{\infty}\left(\mathbf{R}_{+}^{*} \times G\right)$ and satisfy $\partial u(t, x) / \partial t$ $=N u\left(t, x^{-1}\right), \partial w(t, x) / \partial t=N w(t, x)$, respectively, for all $(t, x) \in \mathbf{R}_{+}^{*} \times G$.

Proof. 1. If $p=\infty$, then we have for all $(t, x) \in \mathbf{R}_{+}^{*} \times G$ :

and

$$
u(t, x)=\int f^{*}\left(x^{-1} y\right) \mu_{t}(d y)=\left(T_{\mu_{t}} f^{*}\right)\left(x^{-1}\right)
$$

$$
w(t, x)=\left(T_{\mu_{t}} f\right)(x) .
$$

Hence the assertion in view of Theorem $B$.

2. Now let $p<\infty$ and let $q \in] 1, \infty$ ] be defined by $1 / p+1 / q=1$. Without loss of generality we may assume $f \geqslant 0$. Let $\left(f_{n}\right)_{n \geqslant 1}$ be a sequence in $\mathscr{K}(G)$ such that $\lim \left|f-f_{n}\right|_{p}=0$. Finally we again put $C=\mathbf{R}_{+}^{*} \times G$ and $P=N-\partial / \partial t$.

(a) In view of the proof of the Lemma there exists a function $v \in e^{\infty}(C)$ such that $P v=0, u \leqslant v$ and $u\left(t, x^{-1}\right)=v(t, x)$ for almost all $(t, x) \in C$. In view of Theorem 1(ii) (and of estimate (*) in its proof) the function $t \rightarrow\left|\Delta^{1 / p} v_{t}\right|_{q}$ is finite and, moreover, locally bounded on $\mathbf{R}_{+}^{*}$.

By Hölder's inequality and [1, (20.2)] we have

$$
\left|\mu_{t} * f(x)-\dot{\mu}_{t} * f_{n}(x)\right| \leqslant\left|f-f_{n}\right|_{p}\left|\Delta^{1 / p} v_{t}\right|_{q} \Delta(x)^{-1 / p}
$$

for all $n \geqslant 1$ and $(t, x) \in C$. In view of 1 , every function $(t, x) \rightarrow \mu_{t} * f_{n}(x)$ is continuous. Consequently, the function $(t, x) \rightarrow \mu_{t} * f(x)$ is also continuous. Thus $u\left(t, x^{-1}\right)=v(t, x)$ for all $(t, x) \in C$. Hence the assertion for $u$.

(b) By Hölder's inequality and Theorem 1(i) we have $w(t, x) \leqslant|f|_{p}\left|v_{t}\right|_{q}<\infty$ for all $(t, x) \in C$. Thus the function $w$ is defined and finite. Moreover the estimate

$$
\left|w(t, x)-T_{\mu_{t}} f_{n}(x)\right| \leqslant\left|f-f_{n}\right|_{p}\left|v_{t}\right|_{q}
$$

yields, together with 1 and Theorem 1(ii), the continuity of $w$.

The rest of the proof is again analogous to the proofs of Theorems 1 and 3 in [11]. By

$$
\psi(h)=\int\left(\int w(t, x) h(t, x) \rho(d x)\right) d t \quad(h \in \mathscr{D}(C))
$$

there is defined a distribution $\psi$ on the manifold $C . P$ is a hypoelliptic differential operator on $C$ such that $P \psi=0$. Consequently, there exists a function $v \in C^{\infty}(C)$ 
such that $P v=0$ and $w=v$ almost everywhere. But the continuity of $w$ implies $w=v$. Hence the assertion for $w$.

Let $\pi$ be a strongly continuous representation of $G$ by contractions on a Banach space $E$. For every $\mu \in \Re^{1}(G)$ there is defined a bounded operator $\pi(\mu)$ on $E$ by

$$
\langle\pi(\mu) u, \xi\rangle=\int\langle\pi(x) u, \xi\rangle \mu(d x)
$$

for all $u \in E$ and $\xi \in E^{\prime}$ (where $E^{\prime}$ denotes the strong dual of $E$ and $\langle\cdot, \cdot\rangle$ the canonical bilinear form on $\left.E \times E^{\prime}\right)$. Obviously, $\left(\pi\left(\mu_{t}\right)\right)_{t>0}$ is a strongly continuous semigroup of contractions on $E$. By $\left(N_{\pi}, \Re_{\pi}\right)$ we denote its infinitesimal generator.

THEOREM 3. Let the Banach space $E$ be weakly sequentially complete. Then $\left(\pi\left(\mu_{t}\right)\right)_{t>0}$ is a differentiable operator semigroup, i.e., $\pi\left(\mu_{t}\right) u \in \mathcal{N}_{\pi}$ for all $u \in E$. Moreover, if $u \in E$ and $\xi \in E^{\prime}$ then $\left\langle\pi \pi\left(\mu_{t}\right) u, \xi\right\rangle \in \mathcal{E}(G)$ and

$$
\left\langle N_{\pi}\left(\pi\left(\mu_{t}\right) u\right), \xi\right\rangle=A\left(\left\langle\pi \pi\left(\mu_{t}\right) u, \xi\right\rangle\right) \quad\left(\text { all } t \in \mathbf{R}_{+}^{*}\right) .
$$

Proof. 1. Obviously we have $\langle\pi u, \xi\rangle \in \mathrm{C}^{h}(G)$ and $T_{\mu_{t}}\langle\pi u, \xi\rangle=\left\langle\pi \pi\left(\mu_{t}\right) u, \xi\right\rangle$. Hence in view of Theorem B we have $\left\langle\pi \pi\left(\mu_{t}\right) u, \xi\right\rangle \in \mathcal{E}(G)$. Let $\varphi(\xi)=$ $A\left(\left\langle\pi \pi\left(\mu_{t}\right) u, \xi\right\rangle\right.$ for some fixed $t \in \mathbf{R}_{+}^{*}$ (where $A$ is the generating functional of the semigroup $\left.\left(\mu_{t}\right)_{t>0}\right)$. Let $(t(n))_{n \geqslant 1}$ be some sequence in $\mathbf{R}_{+}^{*}$ converging to 0 . We put

$$
\begin{aligned}
\varphi_{n}(\xi) & =\frac{1}{t(n)}\left\{\left\langle\pi\left(\mu_{t(n)}\right) \pi\left(\mu_{t}\right) u, \xi\right\rangle-\left\langle\pi\left(\mu_{t}\right) u, \xi\right\rangle\right\} \\
& =\frac{1}{t(n)} \int\left\{\left\langle\pi(x) \pi\left(\mu_{t}\right) u, \xi\right\rangle-\left\langle\pi\left(\mu_{t}\right) u, \xi\right\rangle\right\} \mu_{t(n)}(d x) .
\end{aligned}
$$

By definition of $A$ we have

$$
\lim \varphi_{n}(\xi)=\varphi(\xi) \text { for all } \xi \in E^{\prime}
$$

Obviously, every $\varphi_{n}$ is a continuous linear functional on $E^{\prime}$ (defined by the element $t(n)^{-1}\left\{\pi\left(\mu_{t(n)}\right) \pi\left(\mu_{t}\right) u-\pi\left(\mu_{t}\right) u\right\}$ of $\left.E\right)$. By the theorem of Banach and Steinhaus, $\varphi$ is also a continuous linear functional on $E^{\prime}$. Since by our assumption $E$ is weakly sequentially complete there exists an element $v$ of $E$ such that $\varphi(\xi)=\langle v, \xi\rangle$ for all $\xi \in E^{\prime}$.

2. In view of 1 the function $\alpha_{\xi}: s \rightarrow\left\langle\pi\left(\mu_{s}\right) \pi\left(\mu_{t}\right) u, \xi\right\rangle$ (defined on $\mathbf{R}_{+}^{*}$ ) is differentiable from the right and we have

$$
\begin{aligned}
\left.\frac{d^{+}}{d s}\right|_{s=r}\left\langle\pi\left(\mu_{s}\right) \pi\left(\mu_{t}\right) u, \xi\right\rangle & =\left.\frac{d^{+}}{d s}\right|_{s=0}\left\langle\pi\left(\mu_{s}\right) \pi\left(\mu_{t}\right) u, \pi\left(\mu_{r}\right) * \xi\right\rangle \\
& =\varphi\left(\pi\left(\mu_{r}\right)^{*} \xi\right)=\left\langle v, \pi\left(\mu_{r}\right)^{*} \xi\right\rangle=\left\langle\pi\left(\mu_{r}\right) v, \xi\right\rangle
\end{aligned}
$$

(where $\pi\left(\mu_{r}\right)^{*}$ denotes the dual operator of $\pi\left(\mu_{r}\right)$ ). Hence the right derivative of $\alpha_{\xi}$ is continuous. Consequently the function $\alpha_{\xi}$ is continuously differentiable. This yields

$$
\left\langle\pi\left(\mu_{s}\right) \pi\left(\mu_{t}\right) u, \xi\right\rangle-\left\langle\pi\left(\mu_{t}\right) u, \xi\right\rangle=\int_{0}^{s}\left\langle\pi\left(\mu_{r}\right) v, \xi\right\rangle d r
$$

for all $\xi \in E^{\prime}$. Hence

$$
\pi\left(\mu_{s}\right) \pi\left(\mu_{t}\right) u-\pi\left(\mu_{t}\right) u=\int_{0}^{s} \pi\left(\mu_{r}\right) v d r
$$


for all $s \in \mathbf{R}_{+}^{*}$. This yields $\pi\left(\mu_{t}\right) u \in \mathcal{T}_{\pi}$ and $N_{\pi}\left(\pi\left(\mu_{t}\right) u\right)=v$. Consequently we have for all $\xi \in E^{\prime}$ :

$$
\left\langle N_{\pi}\left(\pi\left(\mu_{t}\right) u\right), \xi\right\rangle=\langle v, \xi\rangle=\varphi(\xi)=A\left(\left\langle\pi \pi\left(\mu_{t}\right) u, \xi\right\rangle\right) .
$$

Thus the theorem is proved.

REMARK. Differentiable operator semigroups have been studied, for example, by A. Pazy [8].

Every semireflexive Banach space and every $A L$-space is weakly sequentially complete. (This is obvious for semireflexive spaces; for $A L$-spaces cf. [9, II.8, Corollary of Proposition 8.8].) Hence, Theorem 3 applies in particular to the left regular representation $\pi_{p}$ of $G$ on $L^{p}(G)$ for $1 \leqslant p<\infty$. In this case we have $\pi_{p}(\mu) f=\mu * f$ for all $\mu \in \Re^{1}(G)$ and $f \in L^{p}(G)$. Let $\left(N_{p}, \Re_{p}\right)$ denote the infinitesimal generator of the operator semigroup $\left(\pi_{p}\left(\mu_{t}\right)\right)_{t>0}$ on $L^{p}(G)$.

Corollary 1. We have $v_{t} \in \mathcal{N}_{p}$ and $\left\langle N_{p} v_{t}, g\right\rangle=A\left(\left\langle\pi_{p} v_{t}, g\right\rangle\right)$ for all $t \in \mathbf{R}_{+}^{*}$, $g \in L^{q}(G)$ and $p \in[1, \infty[$ (where $q$ is defined by $1 / p+1 / q=1$ ).

Proof. By Theorem 1 we have $v_{s} \in L^{p}(G)$ and $\pi_{p}\left(\mu_{t}\right) v_{s}=\mu_{t} * v_{s}=v_{t} * v_{s}=v_{t+s}$ for all $s \in \mathbf{R}_{+}^{*}$. Theorem 3 applied to $E=L^{p}(G), \pi=\pi_{p}$ and $u=v_{s}$. $\xi=g$ yields the assertion.

The Banach space $L^{\infty}(G)$ is not weakly sequentially complete. But we can establish a result similar to that for the spaces $L^{p}(G), p<\infty$. For this reason let $\bigodot_{l u}(G)$ (resp. $\bigodot_{r u}(G)$ ) denote the Banach space (with respect to the norm $|\cdot|_{\infty}$ ) of all functions in $e^{b}(G)$ that are uniformly continuous with respect to the left (resp. right) uniform structure on $G$. Then by $T_{t} f(x)=\int f(x y) \mu_{t}(d y), f \in \mathcal{C}_{l u}(G)$ (resp. by $\left.S_{t} f=\mu_{t} * f, f \in \mathcal{C}_{r u}(G)\right)$, there is given a strongly continuous semigroup $\left(T_{t}\right)_{t>0}$ (resp. $\left.\left(S_{t}\right)_{t>0}\right)$ of contractions on $\bigodot_{l u}(G)$ (resp. $\bigodot_{r u}(G)$ ). We denote its infinitesimal generator by $\left(N_{l u}, \Re_{l u}\right)$ (resp. by $\left(N_{r u}, \Re_{r u}\right)$ ).

Corollary 2. For all $g \in L^{\infty}(G)$ and $t \in \mathbf{R}_{+}^{*}$ the following assertions are valid:

(i) $T_{t} g \in \mathcal{K}_{l u}$ and $N_{l u}\left(T_{t} g\right)=\left(N_{1} v_{t} * g^{*}\right)^{*}$.

(ii) $\mu_{t} * g \in \mathcal{K}_{r u}$ and $N_{r u}\left(\mu_{t} * g\right)=\left(N_{1} v_{t}\right) * g$.

Proof. Let $g \in L^{\infty}(G)$ and $t \in \mathbf{R}_{+}^{*}$ be fixed. In view of $\mu_{t} * g=\left(T_{t} g^{*}\right)^{*}$ it suffices to prove (ii). First of all we observe $\mu_{t} * g=v_{t} * g \in \bigodot_{r u}(G)[1,(20.16)]$. Furthermore, for all $s \in \mathbf{R}_{+}^{*}$ the following estimation is valid:

$$
\left|s^{-1}\left\{\mu_{s} *\left(\mu_{t} * g\right)-\mu_{t} * g\right\}-\left(N_{1} v_{t}\right) * g\right|_{\infty} \leqslant\left|s^{-1}\left\{\mu_{s} * v_{t}-v_{t}\right\}-N_{1} v_{t}\right|_{1}|g|_{\infty} .
$$

Now Corollary 1 yields the assertions.

\section{REFERENCES} 1963.

1. E. Hewitt and K. A. Ross, Abstract harmonic analysis. I, Springer-Verlag, Berlin and New York,

2. H. Heyer, Probability measures on locally compact groups, Springer-Verlag, Berlin and New York, 1977.

3. A. Hulanicki, Subalgebra of $L_{1}(G)$ associated with Laplacian on a Lie group, Colloq. Math. 31 (1974), 259-287.

4. P. E. T. Jørgensen, Representations of differential operators on a Lie group, J. Funct. Anal. 20 (1975), 105-135. 
5. J. Kisyński, Holomorphicity of semigroups of operators generated by sublaplacians on Lie groups, Function Theoretic Methods for Partial Differential Equations (Proc. Internat. Sympos., Darmstadt, 1976), Lecture Notes in Math., Vol. 561, Springer-Verlag, Berlin and New York, 1976, pp. 283-297.

6. , On semigroups generated by differential operators on Lie groups, J. Funct. Anal. 31 (1979), 234-244.

7. E. Nelson, Analytic vectors, Ann. of Math. (2) 70 (1959), 572-615.

8. A. Pazy, On the differentiability and compactness of semigroups of linear operators, J. Math. Mech. 17 (1968), 1131-1141.

9. H. H. Schaefer, Banach lattices and positive operators, Springer-Verlag, Berlin and New York, 1974.

10. E. Siebert, Supports of holomorphic convolution semigroups and densities of symmetric convolution semigroups on a locally compact group, Arch. Math. 36 (1981), 423-433.

11. Absolute continuity, singularity, and supports of Gauss semigroups on a Lie group, Monatsh. Math. 93 (1982), 239-253.

12. __ Holomorphy of convolution semigroups, Proc. 7th Conf. on Probability Measures on Groups, Oberwolfach, 1983 (to appear).

Mathematisches Institut, Universität TÚbingen, AUf Der MORgenstelle 10, D-7400 TÚbingen 1, Bundesrepublik Deutschland, Federal Republic of Germany 